\title{
Association between Serum Calprotectin Expression and Disease Activity in Patients with Inflammatory Bowel Disease
}

\author{
R. D. CHEN, YUN YUN HU, QIAN CHEN, HAN XU AND M. J. QIAN* \\ Department of Gastroenterology, The Second Affiliated Hospital of Soochow University, No. 1055 Sanxiang Road, Suzhou, \\ Jiangsu 215000, China
}

Chen et al:: Serum calprotectin expression and correlation with disease activity in IBD patients

\begin{abstract}
Serum calprotectin expression and correlation with disease activity in patients with inflammatory bowel disease is the main objective. 128 patients with inflammatory bowel disease with complete clinical diagnosis and no blood relationship with each other, including 64 patients with Crohn's disease and 64 patients with ulcerative colitis, were collected from January 2018 to January 2020 in our hospital and 64 examiners with normal colonoscopy results were selected as the control group levels. The differences in serum troponin levels between the inflammatory bowel disease group and the normal control group were compared, the differences in serum troponin levels between inflammatory bowel disease patients with different activity levels were compared, the relationship between serum troponin levels and the clinical features of inflammatory bowel disease was analyzed, and finally the diagnostic efficacy of serum troponin levels in inflammatory bowel disease was analyzed. The results showed that serum troponin levels were significantly higher in the inflammatory bowel disease group compared with the control group, but the difference between the Crohn's disease and ulcerative colitis groups was not statistically significant. Singlefactor analysis of variance showed statistically significant $(p<0.05)$ differences in serum troponin levels between Crohn's disease and ulcerative colitis groups in mild activity, moderate activity, severe activity and remission, with severe activity being the highest, followed by moderate activity, mild activity again and remission being the lowest. The specificity of serum calprotectin level in diagnosing inflammatory bowel disease was $97.2 \%$ and the sensitivity was $79.7 \%$; the sensitivity of C-reactive protein test was 73.2 $\%$ and the specificity was $90.8 \%$; the sensitivity of Erythrocyte sedimentation rate test was $65.9 \%$ and the specificity was $86.1 \%$, which was significant. Serum calprotectin levels are associated with the activity of inflammatory bowel disease and may become one of the clinically valuable indicators to determine the activity of inflammatory bowel disease inflammation.
\end{abstract}

Key words: Inflammatory bowel disease, serum calprotectin, disease activity, C-reactive protein, erythrocyte sedimentation rat

Inflammatory bowel disease (IBD), as the sum of a group of chronic intestinal inflammatory diseases, including ulcerative colitis (UC) and Crohn's disease (CD), has not been clearly defined at present ${ }^{[1-3]}$. Its main clinical manifestations are abdominal pain, diarrhoea, mucus, purulent bloody stool, tenesmus, etc. and extraintestinal manifestations such as skin, mucosa, joints, eyes, liver and gallbladder, etc. In recent decades, the incidence rate and incidence rate of IBD in the world have been increasing year by year ${ }^{[4,5]}$. Its pathogenesis is mainly related to environmental and genetic factors, including diet, smoking and infection, which can increase the incidence of IBD. Now a days, IBD has become a common disease of digestive system in China, with high recurrence rate, many complications and high risk of canceration, which seriously affects the quality of life of patients ${ }^{[6,7]}$.

At present, the commonly used diagnosis method of IBD is colonic endoscopy combined with submucosal biopsy, but it belongs to invasive examination, the patient compliance is poor and cannot meet the needs of dynamic observation of the disease. Other examinations such as Computed tomography (CT), Magnetic resonance imaging (MRI) or capsule 
endoscopy cannot be widely used due to the radiation risk and relatively expensive $\cos ^{[8-10]}$. With the in-depth clinical research, various non-invasive examinations have been paid more and more attention by the medical community. The specific biomarkers have been widely paid attention because of the advantages of simple and convenient detection, economy, non-invasive and good patient compliance in the detection and diagnosis of disease activity.

Calprotectin, as a new acute inflammatory marker, has attracted extensive attention of researchers in recent years ${ }^{[11,12]}$. Relevant studies have shown that calprotectin plays an important role in the evaluation of IBD activity, and can be used as an indicator for early diagnosis and judgment of disease activity of IBD. The relationship between calprotectin and clinical diseases, especially with rheumatoid arthritis, cystic fibrosis, systemic lupus erythematosus, colorectal cancer and other diseases has been a large number of studies, but the research on IBD is still lacking. Therefore, it is very important to explore the relationship between calprotectin and IBD. In this study, the levels of Sieric calprotectin (SCP) in different stages of IBD were quantitatively detected by Enzyme-linked immunosorbent assay (ELISA), and the levels of C-reactive protein (CRP) and Erythrocyte sedimentation rate (ESR) were detected at the same time to explore the changes of SCP level in IBD and analyze the relationship between SCP and disease activity, so as to evaluate the clinical value of serum calprotectin in judging the activity of IBD.

\section{MATERIALS AND METHODS}

\section{Clinical data:}

A total of 128 IBD patients with complete clinical diagnosis data and no blood relationship were collected in our hospital from January 2018 to January 2020, including 64 patients with CD and 64 patients with UC. The diagnosis was in accordance with the consensus on diagnosis and treatment of IBD in China.

Inclusion criteria: All patients did not use glucocorticoid therapy, non-steroidal anti-inflammatory drugs (NSAIDs) and Proton pump inhibitor (PPI) preparations in the past $1 \mathrm{mo}$. No immunosuppressant was used in the past $3 \mathrm{mo}$, and there was no clear history of infection in the near future. There were no severe heart disease, lung, nerve and mental disease.

Exclusion criteria: Excluding diabetes, other endocrine and autoimmune diseases, liver and kidney function damage; Women of childbearing age, pregnant women and trauma taking contraceptives. In addition, 64 patients with gastrointestinal symptoms such as abdominal pain and diarrhoea but normal colonoscopy results were selected as the control group. The specific data of IBD group and normal control group are shown in Table 1.

\section{Study methods:}

Blood samples were collected from the median cubital vein of all subjects $12 \mathrm{~h}$ after fasting. The serum was collected in test tubes without pyrogen and endotoxin. After collecting the blood, the serum and red blood cells were separated rapidly and carefully after centrifugation at 3000 for $10 \mathrm{~min}$. The serum samples were frozen at $-20^{\circ}$. Serum calprotectin was detected by ELISA assay, and the detection process was carried out in strict accordance with the instructions of the kit. All reagents and samples were balanced to room temperature before use. According to the operation process of conventional double antibody sandwich ELISA, the absorbance (Optical density (OD) value) of each well was measured in turn with the enzyme labelled instrument set at $450 \mathrm{~nm}$. If the diluted sample with 1:20 volume ratio exceeds the range of standard curve, increase or decrease the dilution ratio until the OD value is within the range of standard curve. CRP was detected by automatic biochemical analyzer (latex enhanced immunoturbidimetric method), ESR was detected by automatic hematocrit detector.

\section{Index of disease activity in patients with IBD:}

ESR, CRP and fecal calprotectin in IBD group were detected by Laboratory of our hospital. The best Clinical Disease Activity Index (CDAI) was used to evaluate the disease activity of $C D$ patients. The remission stage was defined as $\mathrm{CDAI}<150$ and active stage was defined

\begin{tabular}{lccc} 
TABLE 1: GENERAL CLINICAL DATA OF PATIENTS \\
\hline & \multicolumn{2}{c}{ IBD group } & $\begin{array}{c}\text { Control } \\
\text { group }\end{array}$ \\
\cline { 2 - 4 } & CD & UC & \\
Number & 64 & 64 & 64 \\
Gender (male/female) & $37 / 27$ & $31 / 33$ & $35 / 29$ \\
Age (y) & & & \\
$<40$ & 34 & 26 & 28 \\
$\geq 40$ & 30 & 38 & 36 \\
Course of disease (y) & $6.37 \pm 2.56$ & $3.55 \pm 1.57$ & - \\
CDAl activity index & & & \\
Remission period & 15 & 17 & - \\
Mild activity & 14 & 12 & - \\
Moderate activity & 31 & 29 & - \\
Heavy activity & 4 & 6 & - \\
\hline
\end{tabular}


as $\mathrm{CDAI} \geq 150$. Among them, $150-220$ points were mild activity, 221-450 points were moderate activity, and $>450$ points were severe activity. The disease activity score of UC patients was identified by modified Mayo scoring system. The score $\leq 2$ and no single item $>1$ was defined as remission stage, $3-5$ points as mild activity, 6-10 points as moderate activity, and 11-12 points as severe activity.

\section{Statistical analysis:}

Statistical Package for the Social Sciences (SPSS) 20.0 software was used to process the data. The measurement data were expressed by $\mathrm{x} \pm \mathrm{s}$, and normal test and variance " $t$ " test were conducted. The nonnormal distribution was converted into normal distribution by natural logarithm. The count data is expressed by rate. $\mathrm{p}<0.05$ was considered to be statistically significant.

\section{RESULTS AND DISCUSSION}

The serum calprotectin levels in IBD group, CD group and UC group were significantly higher than those in normal control group $(p<0.05)$. There was no significant difference in serum calprotectin level between $C D$ group and UC group ( $\mathrm{p}>0.05)$, as shown in fig. 1 .

The serum calprotectin level of active patients in CD group was $909.27 \pm 278.20 \mathrm{ng} / \mathrm{ml}$, and that of remission patients was $695.45 \pm 207.68 \mathrm{ng} / \mathrm{ml}$, the difference was not statistically significant ( $\mathrm{p}>0.05$ ) (fig. 2). As shown in Table 2, there were significant differences in serum calprotectin levels among mild, moderate, severe and remission groups $(\mathrm{F}=3.087, \mathrm{p}=0.031)$.

In UC group, the serum calprotectin level was $868.31 \pm 375.25 \mathrm{ng} / \mathrm{ml}$ in active phase and 701.58 $\pm 278.88 \mathrm{ng} / \mathrm{ml}$ in remission stage, the difference was statistically significant $(\mathrm{p}<0.05)$, (fig. 3). Univariate Analysis of Variance (ANOVA) analysis showed that there were significant differences in serum calprotectin

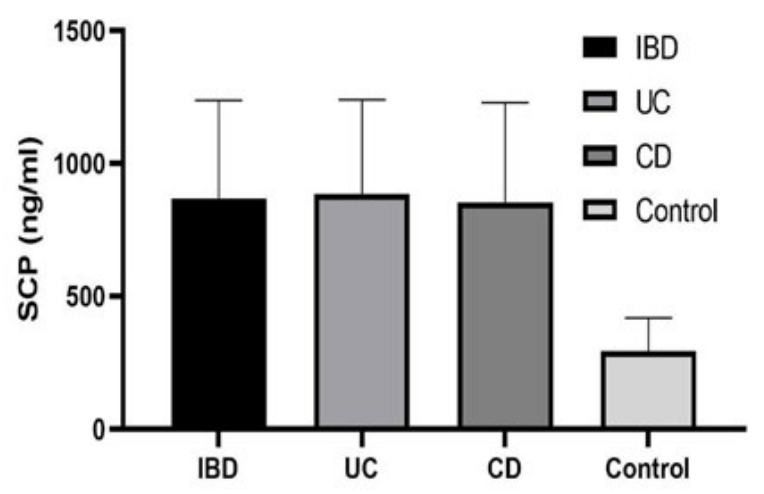

Fig. 1: The expression level of serum calprotectin

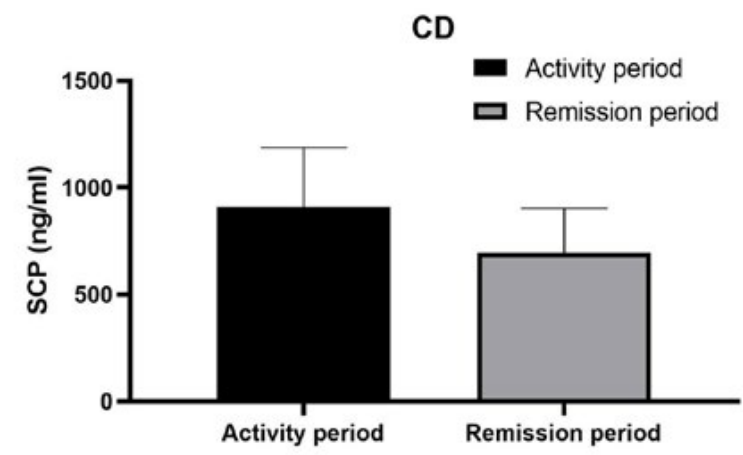

Fig. 2: Serum calprotectin levels of patients with different activity levels were in $\mathrm{CD}$ group

TABLE2: COMPARISONOF SERUM CALPROTECTIN LEVELS IN DIFFERENT ACTIVE AND REMISSION STAGES IN CD GROUP

\begin{tabular}{lcccc}
\hline Group & N & SCP $(\mathrm{ng} / \mathrm{ml})$ & F value & P \\
\hline Heavy activity & 4 & $1218.80 \pm 340.61$ & & \\
Moderate activity & 31 & $937.50 \pm 301.27$ & & \\
Mild activity & 14 & $825.98 \pm 247.26$ & 3.087 & 0.031 \\
Remission period & 15 & $695.45 \pm 207.68$ & & \\
\hline
\end{tabular}

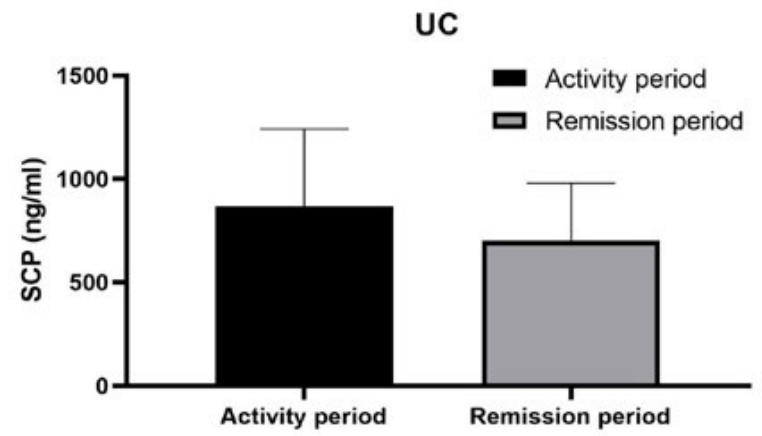

Fig. 3: Serum calprotectin levels in patients with different activity levels in UC group

levels among mild activity, moderate activity, severe activity and remission stage $(\mathrm{F}=3.115, \mathrm{p}=0.025)$. Severe activity was the highest, moderate activity was the second, mild activity was the third, and remission stage was the lowest, as shown in Table 3.

Compared with remission stage, the levels of SCP in mild, moderate and severe active groups were higher $(p<0.01)$. There were significant differences in SCP and CRP between different stages of IBD activity group ( $\mathrm{p}<0.01)$, and the levels of SCP and CRP were gradually increased with the increase of grade. There was no significant difference in CRP level between remission group and mild activity group, and ESR level between mild and moderate active IBD group ( $p>0.05)$, as shown in fig. 4.

The specificity and sensitivity of serum calprotectin level in diagnosing IBD were $97.2 \%$ and $79.7 \%$, 
respectively. The specificity and sensitivity of serum calprotectin level in diagnosing CD and UC were 87.3 $\%$ and $89.7 \%$, respectively, and $98.5 \%$ and $75.6 \%$ respectively. The sensitivity and specificity of CRP were $73.2 \%$ and $90.8 \%$, respectively; the sensitivity and specificity of ESR were $65.9 \%$ and $86.1 \%$ (fig. 5). The sensitivity and specificity of serum calprotectin were the highest, indicating that serum calprotectin has higher sensitivity and specificity in judging the activity of IBD.

IBD, including $\mathrm{CD}$ and $\mathrm{UC}$, is a chronic non-specific intestinal inflammatory disease. Recurrent IBD seriously affects the quality of life and mental health of patients, and mastering the disease activity can guide the treatment and judge the prognosis. At present, the gold standard for diagnosis of IBD and accurate judgment of disease activity are mainly endoscopy and biopsy. However, as an invasive examination, it is time-consuming, expensive and risky. It is difficult to evaluate the disease activity of patients by repeated endoscopic examination. CRP and ESR were used to evaluate the activity of IBD disease, but the sensitivity and specificity were not high ${ }^{[7-10]}$. Disease activity

TABLE 3: COMPARISONOF SERUM CALPROTECTIN LEVELS IN PATIENTS WITH UC AT DIFFERENT ACTIVE AND REMISSION STAGES

\begin{tabular}{lcccc}
\hline Group & N & SCP $(\mathrm{ng} / \mathrm{ml})$ & F value & P \\
\hline Heavy activity & 6 & $1152.49 \pm 463.90$ & & \\
Moderate activity & 29 & $911.72 \pm 349.38$ & 3.193 & 0.025 \\
Mild activity & 12 & $793.37 \pm 272.66$ & & \\
Remission period & 17 & $701.58 \pm 278.88$ & & \\
\hline
\end{tabular}
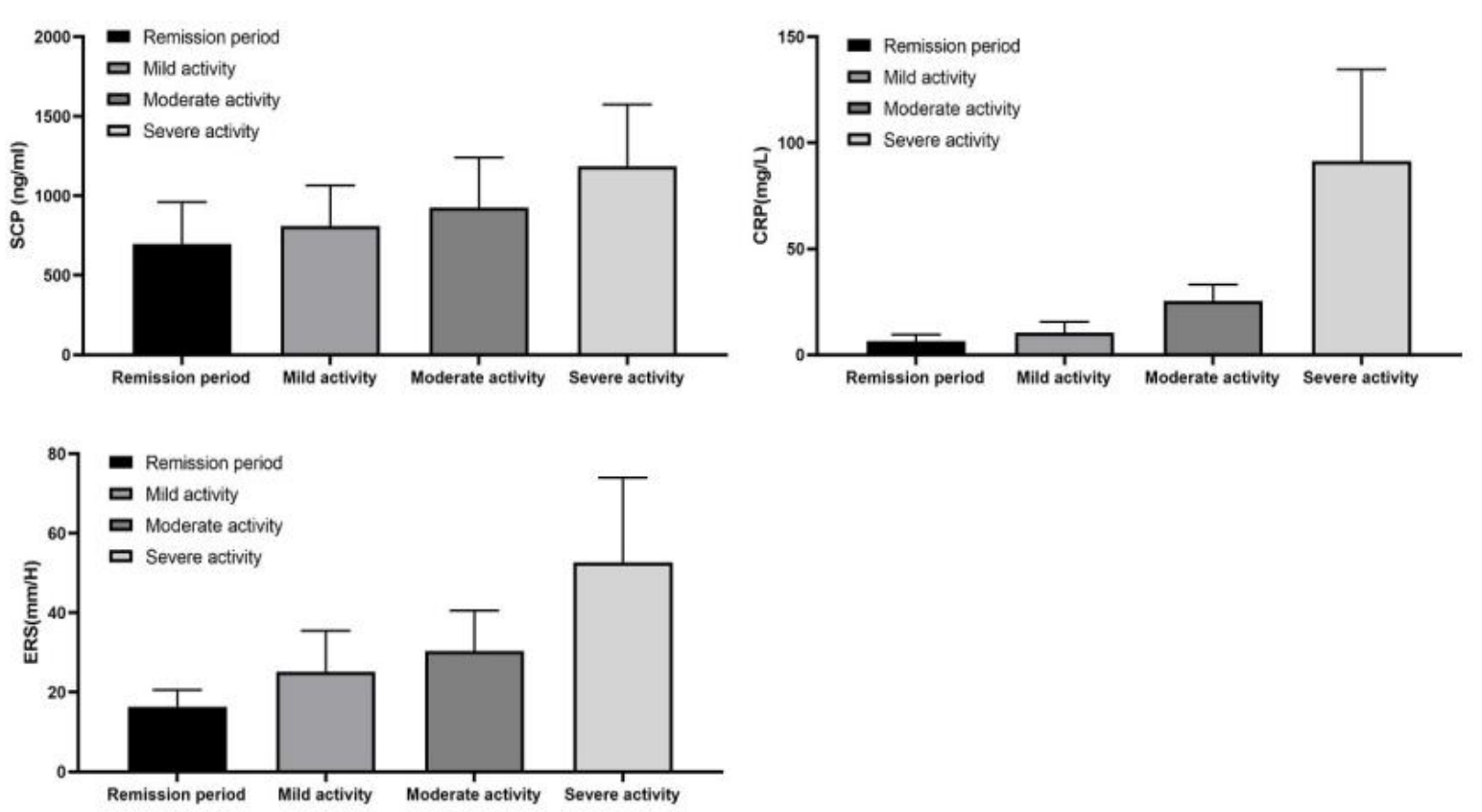

index is also commonly used to evaluate the activity of IBD, but many scoring items depend on the subjective judgment of patients and doctors ${ }^{[6,12]}$. Therefore, a convenient, economical and reliable marker is needed to monitor the activity of IBD. Fecal calprotectin is the most widely used marker for IBD for evaluating intestinal inflammation and mucosal healing. The present study focused on the detection of calprotectin in stool and serum samples, aIso examined the immunohistochemical localization in colon specimens.

Calprotectin is the main protein in neutrophils and macrophages, and it is an inflammatory marker ${ }^{[13]}$. After inflammatory stimulation, neutrophils and macrophages can release calprotectin ${ }^{[14-16]}$. In liver disease and autoimmune disease, calprotectin is significantly related to the severity of the disease, and can predict the prognosis and recurrence of the disease ${ }^{[17-19]}$. When there is inflammation in the gut of IBD patients, a large number of inflammatory cells in the gut, especially neutrophils, can release a large amount of calprotectin into the intestinal cavity mucosa. Therefore, the level of calygin in the intestinal mucosa is related to the inflammation of the gut ${ }^{[20]}$. Numerous studies have shown that the level of fecal calprotectin is related to the intestinal endoscopic performance of IBD patients, which can be used to evaluate the therapeutic efficacy and predict recurrence in IBD patients ${ }^{[21-23]}$. Previous studies have shown that the serum calprotectin level in rats with intestinal inflammation is increased, and it is associated with macro and micro disease activity score $^{[24]}$. Kalla et al. have shown that serum calprotectin 


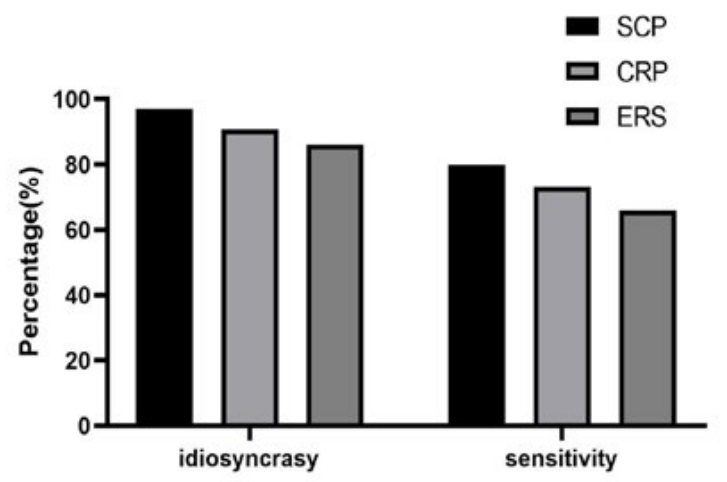

Fig. 5: Diagnostic efficacy of serum calprotectin level for IBD

can be used for the diagnosis of IBD (Area under the curve-AUC $=0.87,95 \%$ CI: 0.78-0.97). Meanwhile, combined with serum calprotectin and CRP or albumin indicators, a scoring system has been established, which can predict the progression of treatment or surgery in patients with $\mathrm{CD}^{[25]}$. In addition, serum calprotectin can predict the recurrence of $\mathrm{CD}$ patients after withdrawal of anti Tumor Necrosis Factor (TNF) drugs ${ }^{[26]}$, and judge the prognosis of patients with severe $\mathrm{UC}^{[27]}$.

In this study, the serum calprotectin level of IBD patients was detected by ELISA, and the levels of CRP, ESR and fecal calprotectin were detected. The disease activity of IBD patients was evaluated by CDAI or Mayo score. We found that the serum calprotectin level of IBD patients was significantly higher than that of normal control group, which was consistent with previous reports. According to the CDAI or Mayo score of IBD patients, the patients were divided into remission stage and active stage, and the active stage was subdivided into mild activity, moderate activity and severe activity. There was no difference in serum calprotectin levels between active and remission patients in $\mathrm{CD}$ group. However, univariate ANOVA analysis showed that there were significant differences in serum calprotectin levels among mild, moderate, severe and remission groups in $\mathrm{CD}$ group. By pairwise comparison, we found that the serum calprotectin level between severe activity and remission stage in $\mathrm{CD}$ group was statistically significant; the serum calprotectin level in UC group was higher than that in remission stage, but there was no significant difference in serum calprotectin level between moderate activity, mild activity and remission stage. At the same time, univariate ANOVA analysis showed that there were significant differences in serum calprotectin levels among mild, moderate, severe and remission groups in UC group. By pairwise comparison, we found that there were significant differences in serum calprotectin levels between severe activity, moderate activity and remission stage in UC group. There are some differences in serum calprotectin levels among different stages of IBD patients, especially in the severe active stage, which indicates that the more severe the intestinal inflammation, the higher the serum calprotectin level.

CRP and ESR are commonly used clinical reference indexes in judging IBD activity. We detected the changes of SCP and CRP in different stages of IBD, and found that compared with remission stage, the levels of SCP in mild, moderate and severe active IBD groups were higher $(\mathrm{p}<0.01)$; there were statistically significant differences in SCP and CRP between different stages of IBD activity group $(\mathrm{p}<0.01)$, and the levels of SCP and CRP were gradually increased with the increase of IBD grade. CRP and ESR had certain sensitivity and specificity (CRP: sensitivity $73.2 \%$, specificity $90.8 \%$; ESR: sensitivity $65.9 \%$, specificity $86.1 \%$ ), significantly lower than SCP. CRP and ESR are less sensitive than SCP, which may be due to the long halflife of CRP, susceptibility to age, anaemia, smoking and other factors, and ESR is susceptible to the influence of cholesterol, globulin and albumin in serum. Therefore, SCP is superior to the first two blood indexes in judging IBD activity, and has higher intestinal sensitivity and specificity.

In conclusion, the activity of IBD can be judged by serum calprotectin level, which has the characteristics of economy, convenience and less pain. For IBD patients who refuse to re-examine enteroscopy repeatedly, the activity of IBD can be preliminarily evaluated by detecting serum calprotectin level. Further exploration and verification can be applied to clinical application.

\section{Acknowledgement:}

This work was supported by The Second Affiliated Hospital of Soochow University, Rui Dong Chen and Yun Yun $\mathrm{Hu}$ contributed equally to this work.

\section{Conflict of Interests:}

The authors declared no conflict of interest.

\section{REFERENCES}

1. Molodecky NA, Soon S, Rabi DM, Ghali WA, Ferris M, Chernoff $\mathrm{G}$, et al. Increasing incidence and prevalence of the inflammatory bowel diseases with time, based on systematic review. Gastroenterology 2012;142:46-54.

2. Zhao J, Ng SC, Lei Y, Yi F, Li J, Yu L, et al. First prospective, population-based inflammatory bowel disease incidence study in mainland of China: the emergence of "western" disease. 
Inflamm Bowel Dis 2013;19:1839-45.

3. Hu R, Ouyang Q, Chen X, Chang Y, Bai A, Wnag R, et al. Analysis of the articles of inflammatory bowel disease in the literature China in recent fifteen years. Chin J Gastroenterol 2007;12:74-7.

4. Zeng Z, Zhu Z, Yang Y, Ruan W, Peng X, Su Y, et al. Incidence and clinical characteristics of inflammatory bowel disease in a developed region of Guangdong Province, China: A prospective population-based study. J Gastroenterol Hepatol 2013;28:1148-53.

5. Tremaine WJ. Is indeterminate colitis determinable? Curr Gastroenterol Rep 2012;14:162-5.

6. Blotière PO, Weill A, Ricordeau P, Alla F, Allemand H. Perforations and haemorrhages after colonoscopy in 2010: a study based on comprehensive French health insurance data (SNIIRAM). Clin Res Hepatol Gastroenterol 2014;38:112-7.

7. Lohsiriwat V. Colonoscopic perforation: incidence, risk factors, management and outcome. World J Gastroenterol 2010; $16: 425-30$.

8. Nie XQ, Chen HH, Bian K. Role of inflammatory markers C-reactive protein, high sensitivity C-reactive protein in diabetes and its cardiovascular complications. Chin J Mod Appl Pharm 2011;28:26-30.

9. Darlington GJ, Wilson DR, Lachman LB. Monocyteconditioned medium, interleukin-1, and tumor necrosis factor stimulate the acute phase response in human hepatoma cells in vitro. J Cell Biol 1986;103:787-93.

10. Lewis JD. The utility of biomarkers in the diagnosis and therapy of inflammatory bowel disease. Gastroenterology 2011;140:1817-26.

11. Mosli MH, Zou G, Garg SK, Feagan SG, MacDonald JK, Chande N, et al. C-reactive protein, fecal calprotectin, and stool lactoferrin for detection of endoscopic activity in symptomatic inflammatory bowel disease patients: a systematic review and meta-analysis. Am J Gastroenterol 2015;110:802-19.

12. Meuwis MA, Vernier-Massouille G, Grimaud JC, Bouhnik Y, Laharie D, Piver E, et al. Serum calprotectin as a biomarker for Crohn's disease. J Crohns Colitis 2013;7:e678-83.

13. Solem CA, Loftus EV, Tremaine WJ, Harmsen WS, Zinsmeister AR, Sandborn WJ. Correlation of C-reactive protein with clinical, endoscopic, histologic, and radiographic activity in inflammatory bowel disease. Inflamm Bowel Dis 2005;11:707-12.

14. Fagan EA, Dyck RF, Maton PN, Hodgson HJ, Chadwick VS, Petrie A, et al. Serum levels of C-reactive protein in Crohn's disease and ulcerative colitis. Eur J Clin Invest 1982;12:351-9.

15. Averill MM, Kerkhoff C, Bornfeldt KE. S100A8 and S100A9 in cardiovascular biology and disease. Arterioscler Thromb Vasc Biol 2012;32:223-9.

16. Katano M, Okamoto K, Suematsu N, Kurokawa MS, Nakamura H, Masuko K, et al. Increased expression of S100 calcium binding protein A8 in GM-CSF-stimulated neutrophils leads to the increased expressions of IL-8 and IL-16. Clin Exp Rheumatol 2011;29:768-75.

17. Vaos G, Kostakis ID, Zavras N, Chatzemichael A. The role of calprotectin in pediatric disease. Biomed Res Int 2013:542363.

18. Nisapakultorn K, Ross KF, Herzberg MC. Calprotectin expression inhibits bacterial binding to mucosal epithelial cells. Infect Immun 2001;69:3692-6.

19. Ghavami S, Chitayat S, Hashemi M, Eshraghi M, Chazin WJ, Halayko AJ, et al. S100A8/A9: a Janus-faced molecule in cancer therapy and tumorgenesis. Eur $\mathrm{J}$ Pharmacol 2009;625:73-83.

20. Alempijevic T, Stulic M, Popovic D, Culafic D, Dragasevic S, Milosavljevic T. The role of fecal calprotectin in assessment of hepatic encephalopathy in patients with liver cirrhosis. Acta Gastroenterol Belg 2014;77:302-5.

21. Garcia-Arias M, Pascual-Salcedo D, Ramiro S, Ueberschlag ME, Jermann TM, Cara C, et al. Calprotectin in rheumatoid arthritis. Mol Diagn Ther 2013;17:49-56.

22. Hradsky O, Ohem J, Mitrova K, Durilova M, Kotalova R, Nevoral $\mathrm{J}$, et al. Fecal calprotectin levels in children is more tightly associated with histological than with macroscopic endoscopy findings. Clin Lab 2014;60:1993-2000.

23. Rugtveit J, Brandtzaeg P, Halstensen TS, Fausa O, Scott H. Increased macrophage subset in inflammatory bowel disease: apparent recruitment from peripheral blood monocytes. Gut 1994;35:669-74.

24. D'Haens G, Ferrante M, Vermeire S, Baert F, Noman M, Moortgat $\mathrm{L}$, et al. Fecal calprotectin is a surrogate marker for endoscopic lesions in inflammatory bowel disease. Inflamm Bowel Dis 2012;18:2218-24.

25. Kalla R, Kennedy NA, Ventham NT, Boyapati RK, Adams AT, Nimmo ER, et al. Serum calprotectin: a novel diagnostic and prognostic marker in inflammatory bowel diseases. Am J Gastroenterol 2016;111:1796-805.

26. Schoepfer AM, Beglinger C, Straumann A, Trummler M, Renzulli P, Seibold F. Ulcerative colitis: correlation of the Rachmilewitz endoscopic activity index with fecal calprotectin, clinical activity, C-reactive protein, and blood leukocytes. Inflamm Bowel Dis 2009;15:1851-8.

27. Sipponen T, Savilahti E, Karkkainen P, Kolho KL, Nuutinen H, Turunen U, et al. Fecal calprotectin, lactoferrin, and endoscopic disease activity in monitoring anti-TNF-alpha therapy for Crohn's disease. Inflamm Bowel Dis 2008;14:1392-8.

This is an open access article distributed under the terms of the Creative Commons Attribution-NonCommercial-ShareAlike 3.0 License, which allows others to remix, tweak, and build upon the work non-commercially, as long as the author is credited and the new creations are licensed under the identical terms

This article was originally published in a special issue, "Clinical Research in Pharmaceutical and Biomedical Sciences" Indian J Pharm Sci 2021:83(1)Spl issue1;12-17 